\title{
Road Hazard Assessment Using Pothole and Traffic Data in South Korea
}

\author{
Choong Heon Yang $\mathbb{D}^{D}$, Jin Guk Kim $\mathbb{D}^{\text {, }}$, and Sung Pil Shin \\ Korea Institute of Civil Engineering and Building Technology, Goyang-si, Ilsanseo-Gu, Gyeonggi-do 10223, Republic of Korea \\ Correspondence should be addressed to Choong Heon Yang; chyang@kict.re.kr
}

Received 19 April 2021; Accepted 21 July 2021; Published 9 August 2021

Academic Editor: Yuchuan Du

Copyright ( $\odot 2021$ Choong Heon Yang et al. This is an open access article distributed under the Creative Commons Attribution License, which permits unrestricted use, distribution, and reproduction in any medium, provided the original work is properly cited.

\begin{abstract}
Road surface conditions have a direct effect on the quality of driving, which in turn affects overall traffic flow. Many studies have been conducted to accurately identify road surface conditions using diverse technologies. However, these previously proposed methods may still be insufficient to estimate actual risks along the roads because the exact road risk levels cannot be determined from only road surface damage data. The actual risk level of the road must be derived by considering both the road surface damage data as well as other factors such as speed. In this study, the road hazard index is proposed using smartphone-obtained pothole and traffic data to represent the level of risk due to road surface conditions. The relevant algorithm and its operating system are developed to produce the estimated index values that are classified into four levels of road risk. This road hazard index can assist road agencies in establishing road maintenance plans and budgets and will allow drivers to minimize the risk of accidents by adjusting their driving speeds in advance of dangerous road conditions. To demonstrate the proposed risk hazard assessment methodology, road hazards were assessed along specific test road sections based on observed pothole and historical travel speed data. It was found that the proposed methodology provides a rational method for improving traffic safety.
\end{abstract}

\section{Introduction}

The various defects that frequently occur on roadways can be easily observed while driving. They generally include potholes, road faults, blow-ups, spalling, and cracks. Among these defects, potholes are likely to be considered the most typical by drivers; thus, they generally cause the most anxiety. Potholes are exacerbated by rain, freezing, and the overuse of deicing materials, especially during winter. They can be very dangerous depending on their dimensions and traffic speed. The more severe the road surface damage, the greater the risk of flat tires and collisions. Therefore, a great deal of effort has been expended to collect accurate information on asphalt pavement conditions using diverse technologies [1-3]. Nevertheless, traffic problems related to potholes have not decreased in recent years in South Korea. Approximately 47,200 potholes were reported from 2015 to 2019 along the $4,767 \mathrm{~km}$ of Korean expressways; this averages to 10 potholes per km. Additionally, 221 pothole-related traffic accidents were reported from 2017 to 2019 [4]. In the Seoul Metropolitan Area, 33,885 potholes were reported, 36.5\% of which occurred during the wet season in July and August [5]. Even if potholes can be rapidly reported, effectively doing so largely relies on active correspondence between drivers and public agency patrols. Thus, by ordinary means, it is highly unlikely that detection alone can be efficient enough to ensure safe roadways.

To address this issue, various automatic detection techniques have been proposed [6-10]. Over the past decade, road surface damage has been shown to be detectable by analyzing the patterns of vehicle vibrations caused by direct contact with the roadway. Such detection can be conducted at low costs and is suitable for real-time assessment but is restricted to the parts of the road touched by the vehicle tires. Thus, the true magnitude of damage to the roadway cannot be assessed [11]. Indirect detection technologies such as laser scanning and image processing have been actively employed 
as a result [12]. These technologies have the ability to detect areas wider than those captured by wheel vibrations. Furthermore, there is a great deal of flexibility regarding the equipment and price of adopting these technologies.

The technology employed in this research therefore collects pothole data using in-vehicle smartphone accelerometers and cameras to obtain images of road surface anomalies $[13,14]$. These images are then analyzed with an image recognition-based fully convolutional neural network model to determine if they contain potholes. The final verified pothole images are then transmitted to a server along with the vehicle's Global Positioning System (GPS) data. The Data Interchange Factory for Safety Solution (DAISS) system, which was developed to provide novel information considering road inventory data, is used for information management in this study $[15,16]$. The novel information provided by this implementation includes road hazards that indicate potentially impeded traffic flow. The main purpose of providing this information is to proactively mitigate the risk of traffic accidents caused by poor road surface conditions. Indeed, traffic accidents are currently among the most serious problems facing our society and thus require ceaseless technological and policy upgrades [17]. From a traffic safety perspective, road hazard information is essential to guide road agencies and general driver decision making. Furthermore, the success of the impending era of autonomous driving depends on such information.

Clearly, even if accurate data describing the locations of potholes are collected, these data are likely to be insufficient to estimate the actual risks along the roads. The DAISS therefore includes a core algorithm that enables the generation of road hazard information in the form of a four-level index indicating the road risk. This road hazard index can better assist road agencies in establishing road maintenance plans and planning for their costs. Furthermore, drivers can minimize the risk of accidents by adjusting their driving speeds in advance of dangerous road conditions indicated by the index.

The most significant advance related to this research is the development of the road hazard index that uses pothole data collected from in-vehicle devices with road inventory data accumulated over a long period of time by the Korean road authorities. To demonstrate the proposed detection method and hazard index, road hazards along a specific test road section were assessed using the observed pothole and historical travel speed data. It is shown that the proposed methodology provides a rational method for improving traffic safety.

\section{Generating the Road Hazard Index}

2.1. Algorithm Description. The algorithm used here is basically referred from previous studies. Pothole data can be detected using the technological system illustrated in Figure 1. The accuracy of this technology has been verified in previous reviews [13, 14, 18]. Using DAISS, point-based detected pothole data can be transformed into a roadway section-based map using the Korean standard transportation node-link system (https://its.go.kr/nodelink/intro). This system was developed to support the smooth exchange of traffic information across Korea and includes a database that applies a unified identification system within the national transportation network.

The road-surface anomalies in the region of interest (ROI) marked with a red box were detected and were marked with yellow boxes in Figure 1. The collected relevant images were used as input for the FCN (fully convolutional network) model to detect road surface anomalies. This is a deep learning network structure for segmentation, and it is a technique that divides the original image by grouping meaningful parts together. In addition, the graph in Figure 1 shows the change of the 3-axis accelerometer built into the smartphone. When a road surface abnormality is detected, they can be measured for 3 seconds. The road hazard index algorithm was developed using pothole data collected from in-vehicle smartphone cameras as well as the average travel speeds across the corresponding roadway sections. The average travel speed was selected as a critical variable based on two considerations: the opinions of 30 transportation professionals in the public and private sectors and the presence or absence of real- and non-real-time data. Real-time information is of practical importance because it can be used in real time by drivers and private traffic information providers. On the other hand, non-real-time information is typically used by road agencies to plan road maintenance. Thus, the algorithm should account for both the degree of road surface damage risk and traffic speed. The relative risk of a given road section in terms of pothole data can thus be expressed as follows:

$$
\text { pothole risk degree }(\mathrm{PRD})=\lambda_{t}^{p d} \times \text { average travel speed }(\mathrm{km} / \mathrm{h}) \text {, }
$$

where $\lambda_{t}^{p d}$ is the pothole detection rate of a vehicle during time $t$, which can be expressed as follows:

$$
\lambda_{t}^{p d}=\frac{C V_{\text {detect }}}{C V_{\text {pass }}},
$$

where $C V_{\text {pass }}$ is the number of vehicles passing a specific road section and $C V_{\text {detect }}$ is the number of vehicles detecting potholes. Only vehicles having the smartphone technology described above are counted. When the real-time average travel speed is used, the pothole risk degree can be considered real time. Otherwise, the pothole risk degree must be considered non-real-time. Figure 2 illustrates the algorithm embedded in DAISS with flowchart. Comparison of the speed limit and the average travel speed of the analyzed road sections is crucial to the process.

2.2. Road Hazard Index. The algorithm shown in Figure 2 was developed to enable the normalization of the detected pothole data by calculating the $Z$ value based on the assumption that the data will have a normal distribution. This calculation is based on the $Z$-value transform given by

$$
Z_{t}^{i}=\left(\frac{X_{t-1, t}-\mu_{0, t}}{\sigma_{0, t}}\right),
$$

where $\mu_{0, t}^{i}$ and $\sigma_{0, t}^{i}$ represent the mean and standard deviation, respectively, of detected pothole information $i$ collected from vehicles during time $t$ and $X_{t-1}^{i}$ is the mean of detected pothole information $i$ collected from vehicles 


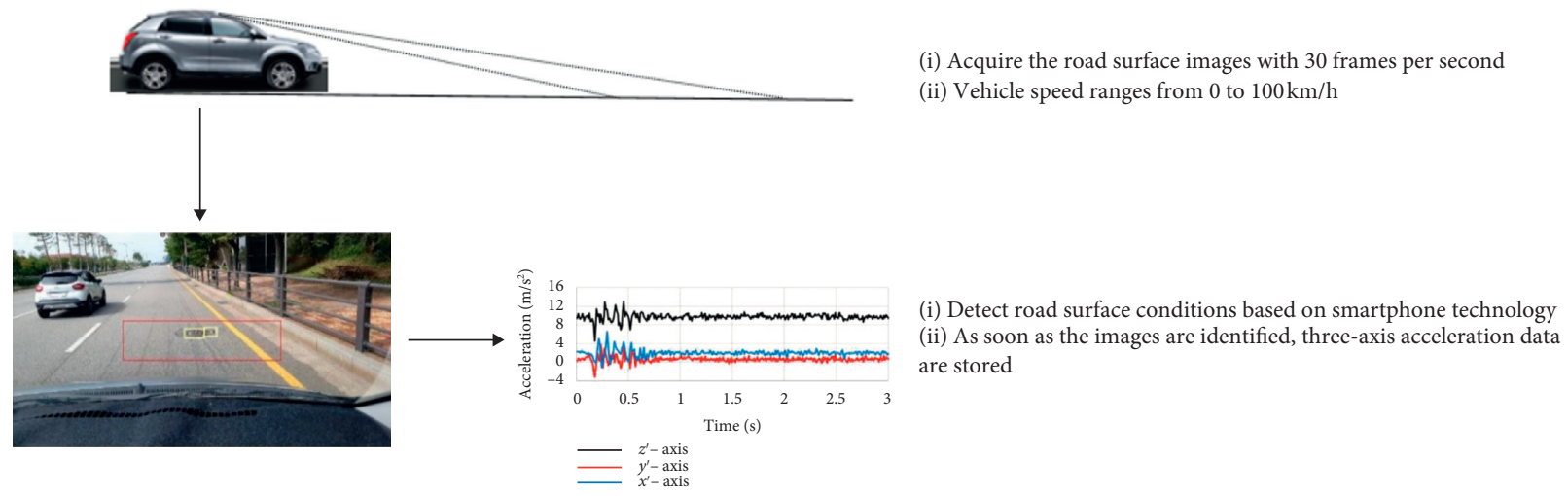

Figure 1: Overall flow of image and three-axis acceleration data acquisition using a smartphone camera.

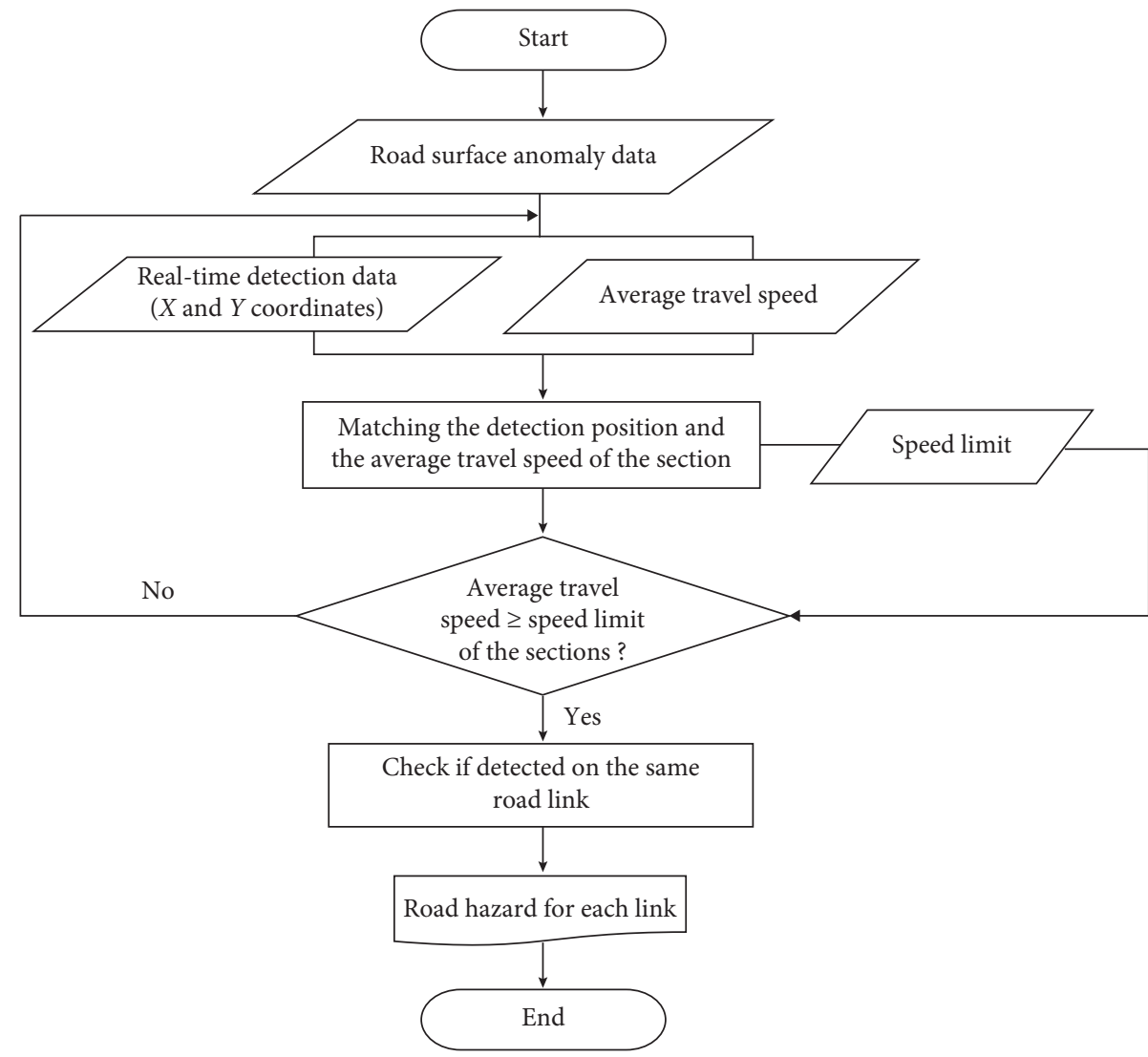

FIgURE 2: Algorithm processing flow in DAISS.

between $t-1$ and $t$. Generally, 30 samples (i.e., 30 vehicles passing a specific roadway section) are sufficient to ensure the statistical validity of such information; therefore, at least 30 samples are required to approximate a normal distribution. Thus, after the PRD values are generated, they can be normalized using the above equation. Then, the normalized values can be converted into specific scores using the conversion equations shown in Table 1 . These equations correspond to the normalized $Z$ values in a series of intervals to define a 100 -point scale $[19,20]$. The final conversion score will allow users to intuitively grasp the risk levels associated with road surface damage.
In Table 1 , the minimum score was set to 50 to prevent the conversion scores from being excessively affected by an abnormally low $Z$ value. Consequently, an interval scale ranging from 50 to 100 was established based on the converted scores. Also, it is a fact that public agencies prefer a tight scale to a wide one. This is because it is possible to make quick decisions about the level of road risk based on the score. The four hazard levels presented in Table 2 (i.e., caution, alert, warning, and danger) were taken from the classification scale found in the "Stage-Specific Road Emergency Response Manual (Heavy Snowfall)” currently used by the Korean government. 
TABLE 1: Equations for converting the road conditions into a 100point scale.

\begin{tabular}{lc}
\hline Condition & Conversion equations \\
\hline $1.645 \leq Z \leq \operatorname{Max}(Z)$ & $1.645)\}] \times 0.5\}$ \\
& $\{[(95+5 \times\{(Z-1.645) /(\mathrm{Max}(Z)-$ \\
$1.282 \leq Z \leq 1.645$ & $(1.645-1.282)\}] \times 0.5\}$ \\
& $50+\{[(85+5 \times\{(Z-1.038) /$ \\
$1.038 \leq Z \leq 1.282$ & $(1.282-1.038)\}] \times 0.5\}$ \\
& $50+\{[(80+5 \times\{(Z-0.842) /$ \\
$0.842 \leq Z \leq 1.038$ & $(1.038-0.842)\}] \times 0.5\}$ \\
& $50+\{[(75+5 \times\{(Z-0.676) /$ \\
$0.676 \leq Z \leq 0.842$ & $(0.842-0.676)\}] \times 0.5\}$ \\
& $50+\{[(70+5 \times\{(Z-0.526) /$ \\
$0.526 \leq Z \leq 0.676$ & $(0.676-0.526)\}] \times 0.5\}$ \\
& $50+\{[(65+5 \times\{(Z-0.387) /$ \\
$0.387 \leq Z \leq 0.526$ & $(0.526-0.387)\}] \times 0.5\}$ \\
& $50+\{[(60+5 \times\{(Z-0.255) /$ \\
$0.255 \leq Z \leq 0.387$ & $(0.387-0.255)\}] \times 0.5\}$ \\
& $50+\{[(40+5 \times\{(Z+0.255) /$ \\
$-0.255 \leq Z \leq 0.255$ & $(0.255+0.255)\}] \times 0.5\}$ \\
& $50+\{[(35+5 \times\{(Z+0.387) /$ \\
$-0.387 \leq Z \leq-0.255$ & $-0.255+0.387)\}] \times 0.5\}$ \\
& $50+\{[(30+5 \times\{(Z+0.526) /$ \\
$-0.526 \leq Z \leq-0.387$ & $(-0.387+0.526)\}] \times 0.5\}$ \\
& $50+\{[(25+5 \times\{(Z+0.676) /$ \\
$-0.676 \leq Z \leq-0.526$ & $(-0.676+0.842)\}] \times 0.5\}$ \\
& $50+\{[(20+5 \times\{(Z+0.842) /$ \\
$-0.842 \leq Z \leq-0.676$ & $(-0.676+0.842)\}] \times 0.5\}$ \\
& $50+\{[(15+5 \times\{(Z+1.038) /$ \\
$-1.038 \leq Z \leq-0.842$ & $(-0.842+1.038)\}] \times 0.5\}$ \\
$-1.282 \leq Z \leq-1.038$ & $50+\{[(10+5 \times\{(Z+1.282) /$ \\
& $(-1.038+1.282)\}] \times 0.5\}$ \\
$-1.645 \leq Z \leq-1.282$ & $(-1.282+1.645)\}] \times 0.5\}$ \\
Min $(Z) \leq$ & $\{(0+5 \times\{(Z+\mathrm{Min}(Z)) /$ \\
$Z \leq-1.645$ & $(-1.645+\mathrm{Min}(Z))\}] \times 0.5\}$ \\
&
\end{tabular}

TABLE 2: Road hazard index.

\begin{tabular}{lcccc}
\hline Category & Caution & Alert & Warning & Danger \\
\hline Score out of 100 & $50 \sim 63$ & $64 \sim 75$ & $76 \sim 88$ & $89 \sim 100$ \\
\hline
\end{tabular}

2.3. DAISS Overview. Informatization of data in Korea began in 1978 with the computerization of government projects through a system that remains in use today. Various government-led programs for nationwide informatization have been planned and implemented since then [21]. In particular, the Ministry of Land, Infrastructure and Transport has strengthened the fusion of national geospatial information and has built and operates an informationtechnology- (IT-) based information sharing system. Figure 3 depicts the fundamental concept underlying DAISS, which consists of three process steps:

Step 1. Pothole data detected by in-vehicle smartphone technology are directly connected to DAISS.

Step 2. Pothole data are combined with travel speed data from other databases using the algorithm in DAISS.
Step 3. The road hazard index is generated based on the roadway section.

The generated road hazard index can be provided to the private and public sectors via DAISS, which is configured to quantify road conditions for transmission to road agencies and users in various formats (e.g., text, index, and visualizations). Furthermore, the method of information sharing applies an Open-API to collect location-based information from GIS [22].

The Highway Management System (HMS) was developed in the early 2000s and has been used for systematic maintenance and management of Korean national highways with high efficiency through support by data-based analysis [23]. The most important feature of HMS is the informatization of all aspects of road management, including incident prevention and safety (e.g., landslides), snow removal management, road occupancy, problem reporting, bridge and tunnel status, and traffic speed and volume. All databases accumulated in the HMS include non-real-time data. These databases are not open to the public because they are intended to be used only for road maintenance purposes. Figure 4 illustrates the DAISS development results using the road hazard algorithm and its corresponding index, as well as embedded features provided for user convenience [18].

\section{Field Application}

This section presents the results of an assessment of actual road surface conditions based on pothole and HMS data collected from test roads in Daegu and Gwangju cities at the end of November 2020. A total of 30 vehicles were employed in Daegu and 65 vehicles were employed in Gwangju. These cities were selected because they already possess a large quantity of pothole data, and their historical travel speed databases are well organized. After the road hazard index was generated, two important tasks were undertaken to confirm the reliability of the analysis results. First, a field survey was performed in some of the data collection sections in order to verify the agreement between the index and actual road surface conditions, as shown in Figure 5. Second, in-person interviews were conducted with the road agencies in charge of the respective roadway sections.

Figures 6 and 7 show the road hazard index values obtained for Gwangju and Daegu cities, respectively. During the test period (i.e., December 2020), a daily average of 140 detection data points was linked to DAISS. The road hazard index results were generated by applying the algorithm based on the detection data and the average travel speeds as recorded in the HMS.

In the test roadway sections of Gwangju, the index generally ranged between 50 and 63 , corresponding to the "caution" level (green color), and only four of 30 test sections fell within the range of 64-75, corresponding to the "alert" level (yellow color). Reliability was confirmed using data from the field survey and interviews with public agencies. The index values for Daegu clearly differed from those for Gwangju. This is likely a result of the different road and traffic conditions in the two cities. Because the test roads in 

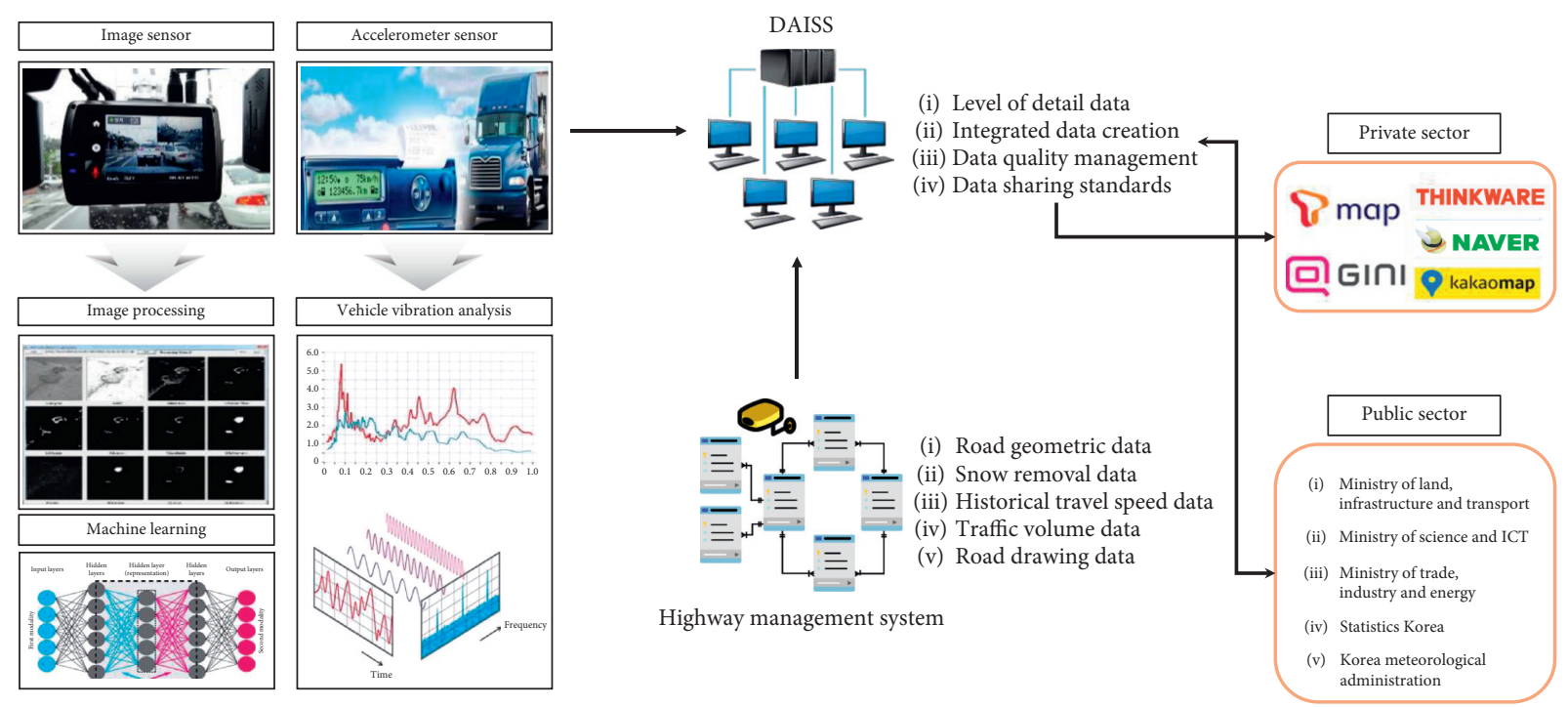

FIgure 3: Concept of DAISS.

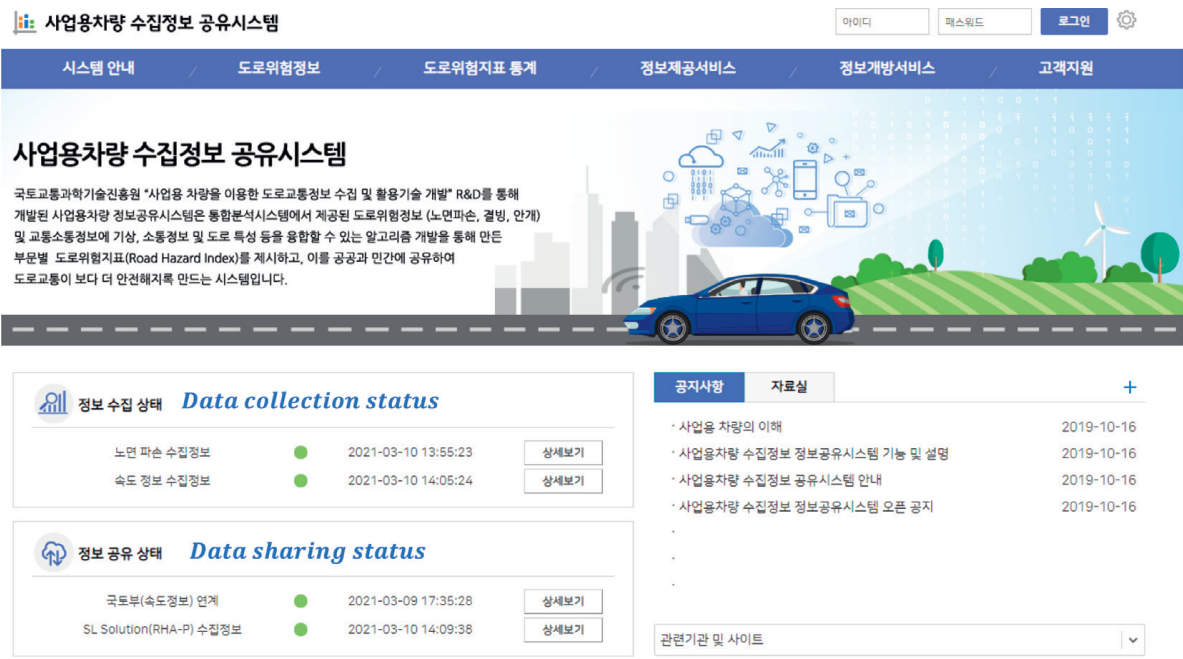

(a)

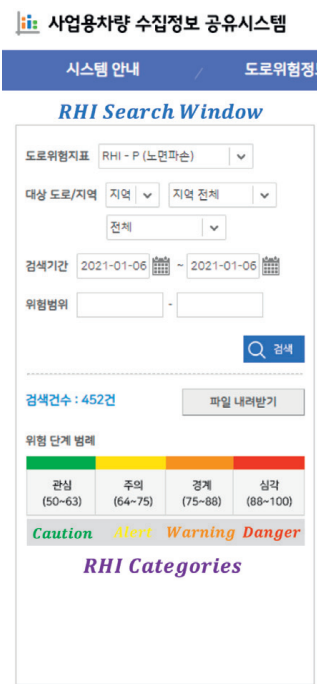

\begin{tabular}{|c|c|c|}
\hline $0 ;|0| 1 \mid$ & 패스으드 & 로그민 \{ \\
\hline & & 고과 \\
\hline
\end{tabular}

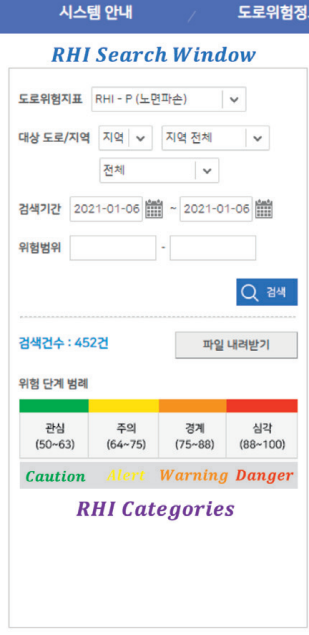

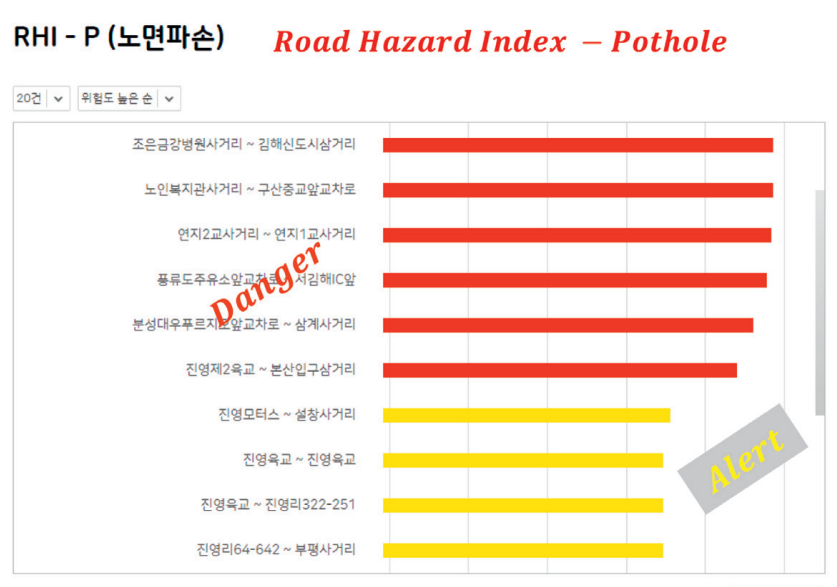

(b)

Figure 4: Continued. 


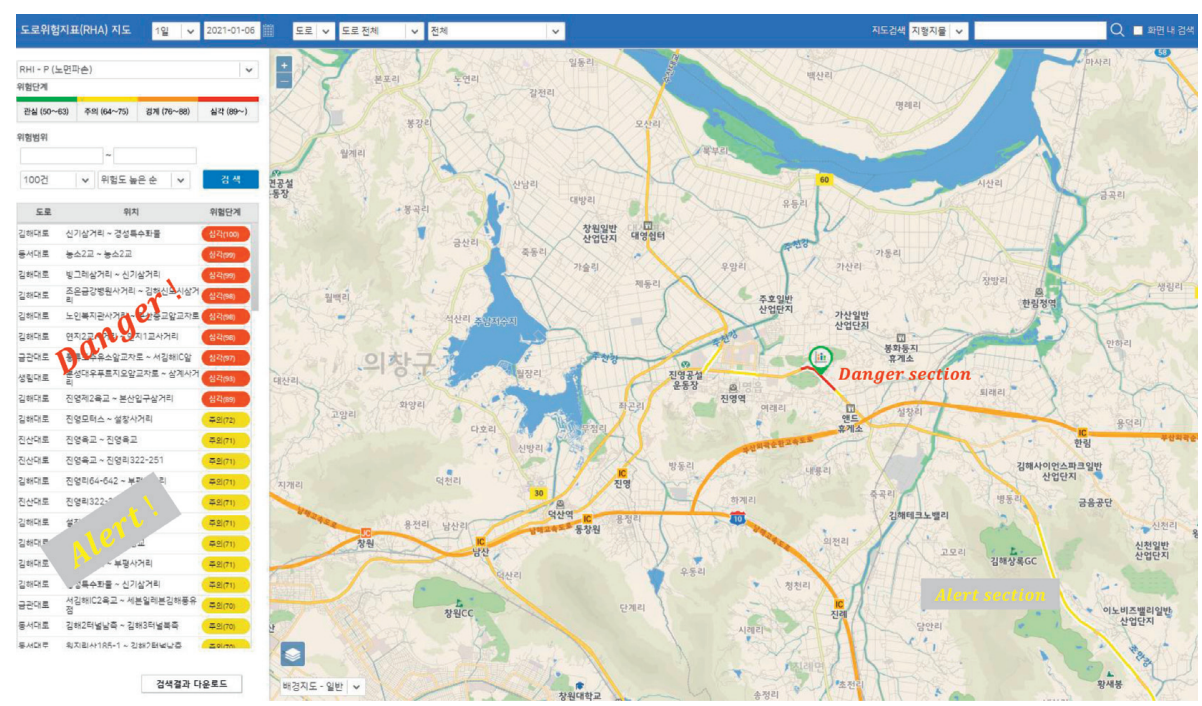

(c)

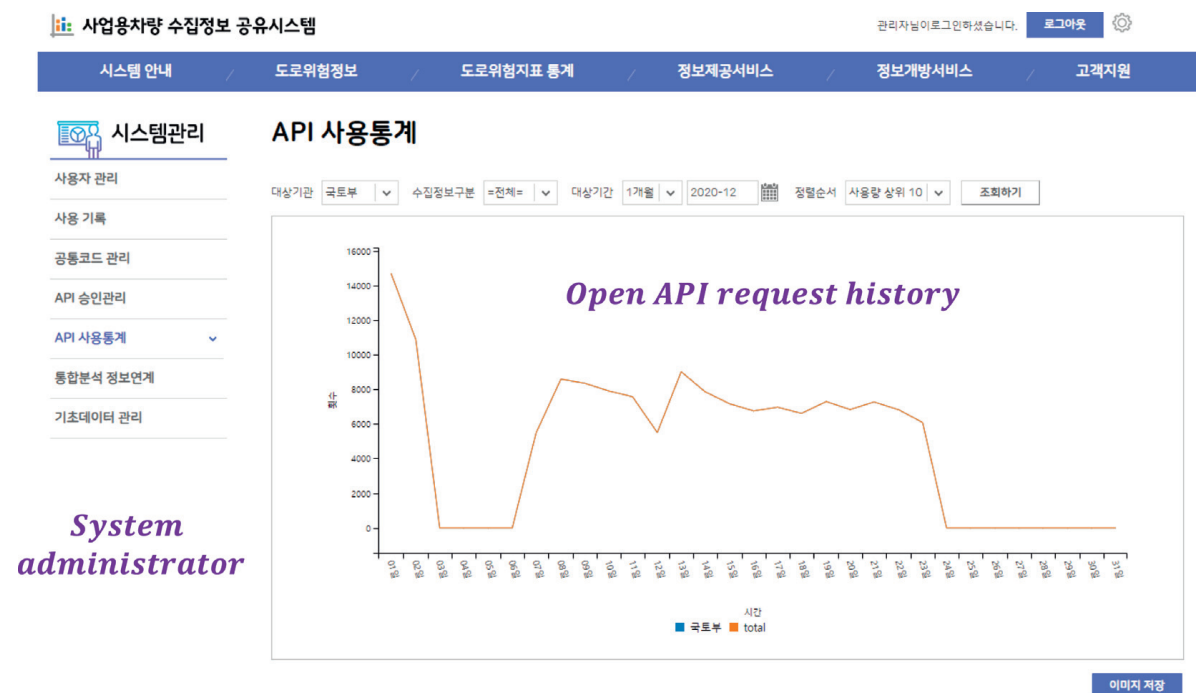

(d)

FIgURE 4: DAISS development results. (a) Main menu. (b) Road hazard index statistics. (c) GIS map-based information platform. (d) DAISS usage statistics.

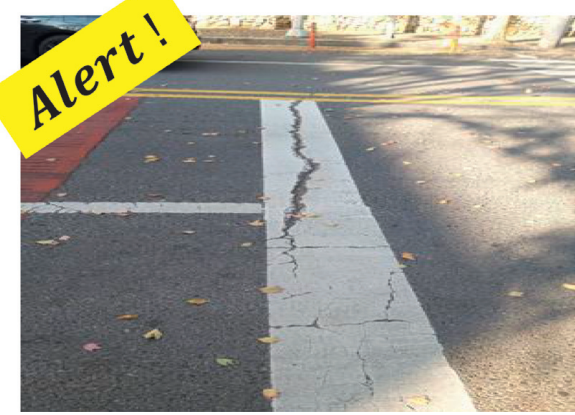

(a)

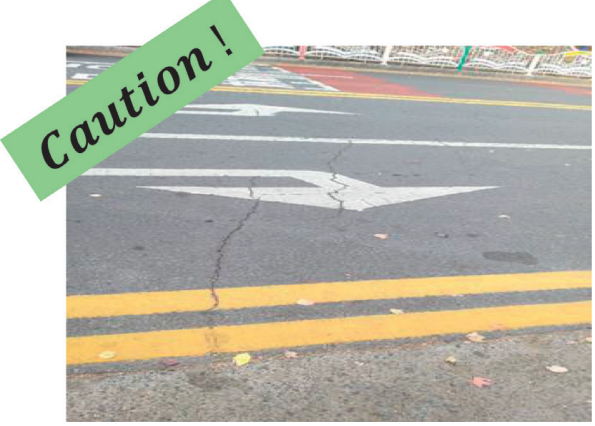

(b)

Figure 5: Field survey and analysis results in Gwangju and Daegu cities. (a) Gwangju. (b) Daegu. 


\begin{tabular}{|c|c|c|c|c|c|c|c|c|c|c|c|}
\hline No & Date & Link ID & Section & $\lambda_{\mathrm{pd}}$ & $C V_{\text {pastin }}$ & $C V_{\text {ponotiodater }}$ & $\lambda_{\text {pd }} \times$ traffic_volume & FRD & $z$ & RHI-P & RHI-Index \\
\hline 1 & 20201210 & $\begin{array}{l}7750002900 \\
\end{array}$ & Nammunro & 0.3333 & 3 & 1 & 17032 & $\begin{array}{l}17032 \\
\end{array}$ & 2.6667 & 97.9325 & 4 \\
\hline 2 & 20201210 & 1750002300 & $\begin{array}{l}\text { Nammunro } \\
\end{array}$ & 0.3333 & 3 & 1 & 17032 & 17032 & & 97.8513 & \\
\hline 3 & 20201210 & & $\begin{array}{l}\text { Nammunro } \\
\end{array}$ & & 1 & 1 & 51096 & 51096 & 2.1963 & 977.7334 & \\
\hline 4 & 20201210 & 1750004202 & $\begin{array}{l}\text { Pilmundaero } \\
\end{array}$ & 1 & 1 & 1 & 13032 & 13032 & 1.7928 & 97.5626 & 4 \\
\hline 5 & 20201210 & 1790204900 & Udongdaero & 0.5 & 2 & 1 & 11673 & 11673 & 1.7889 & 97.5609 & 4 \\
\hline 6 & 20201210 & 1750004702 & Pilmundaero & 1 & & & 13032 & 13032 & 1.557 & 96.8939 & 4 \\
\hline 7 & 2020121 & 1750004300 & Pilmundaero & 1 & & & 13 & 130 & 1.3333 & 95.3535 & \\
\hline 8 & 20201210 & 175000 & Pilmundaero & 1 & 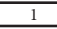 & 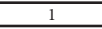 & 13 & 13032 & 1.2613 & 94.788 & 4 \\
\hline 9 & 20201210 & 1750004 & Pilmundaero & 1 & 1 & 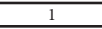 & 130 & 13032 & 1.1866 & 27 & 4 \\
\hline 10 & 20201210 & 1750002502 & Nammunro & 0.25 & 4 & 1 & 12774 & 12774 & 0.9428 & 1.2858 & 4 \\
\hline 11 & 20201210 & 1750003400 & Daenamdaero & 0.5 & 2 & 1 & 25548 & 25548 & 0.7263 & 88.2571 & 3 \\
\hline 12 & 20201210 & 1750002700 & Nammunro & 0.3333 & 3 & 1 & 17032 & 17032 & 0.378 & 82.3289 & 3 \\
\hline 13 & 20201210 & 1790008402 & $\begin{array}{l}\text { Udongdaero } \\
\end{array}$ & 0 & 2 & 0 & 0 & 0 & -0.2774 & 699.5767 & 2 \\
\hline 14 & 20201210 & 1750001900 & $\begin{array}{l}\text { Nammunro } \\
\end{array}$ & 0 & -1 & 0 & 0 & 0 & -0.3333 & 68.5164 & 2 \\
\hline 15 & 20201210 & 1790008503 & Udongdaero & 0 & 3 & 0 & 0 & 0 & -0.3333 & 68.5164 & 2 \\
\hline 16 & 20201210 & 1790201900 & Udongdaero & 0 & 1 & 0 & 0 & 0 & -0.3536 & 68.1335 & 2 \\
\hline 17 & 20201210 & 17500040 & Pilmundaero & 0 & 1 & 0 & 0 & 0 & -0.4082 & 67.1178 & $\frac{2}{2}$ \\
\hline 18 & 20201210 & 175000 & Pilmundaero & 0 & 1 & 0 & 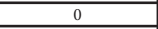 & 0 & -0.4472 & 66.417 & 2 \\
\hline 19 & 20201210 & 175000 & Pilmundaero & 0 & 3 & 0 & 0 & 0 & $\begin{array}{l}-0.4743 \\
\end{array}$ & 655.9291 & 2 \\
\hline 20 & 20201210 & 1790203600 & $\begin{array}{l}\text { Donggokro } \\
\end{array}$ & 0 & 1 & & 0 & - & -0.5 & 5.4676 & 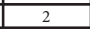 \\
\hline 21 & 2020121 & 1790250800 & $\begin{array}{l}\text { Donggokro } \\
\end{array}$ & 0 & 1 & 7 & - & 0 & 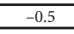 & 65 & 2 \\
\hline 22 & 2020 & 17902717 & & 0 & 1 & 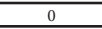 & 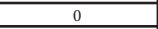 & 0 & -1 & & 2 \\
\hline 23 & 20201210 & 1790008502 & & 0 & 3 & 0 & 0 & 0 & -0.6211 & 63.4157 & 1 \\
\hline 24 & 20201210 & 15400 & bukro & 0 & & 0 & 0 & 0 & 0 & 50 & - \\
\hline & 20201210 & 1750001300 & Nammunro & & & & & & & & \\
\hline
\end{tabular}

(a)

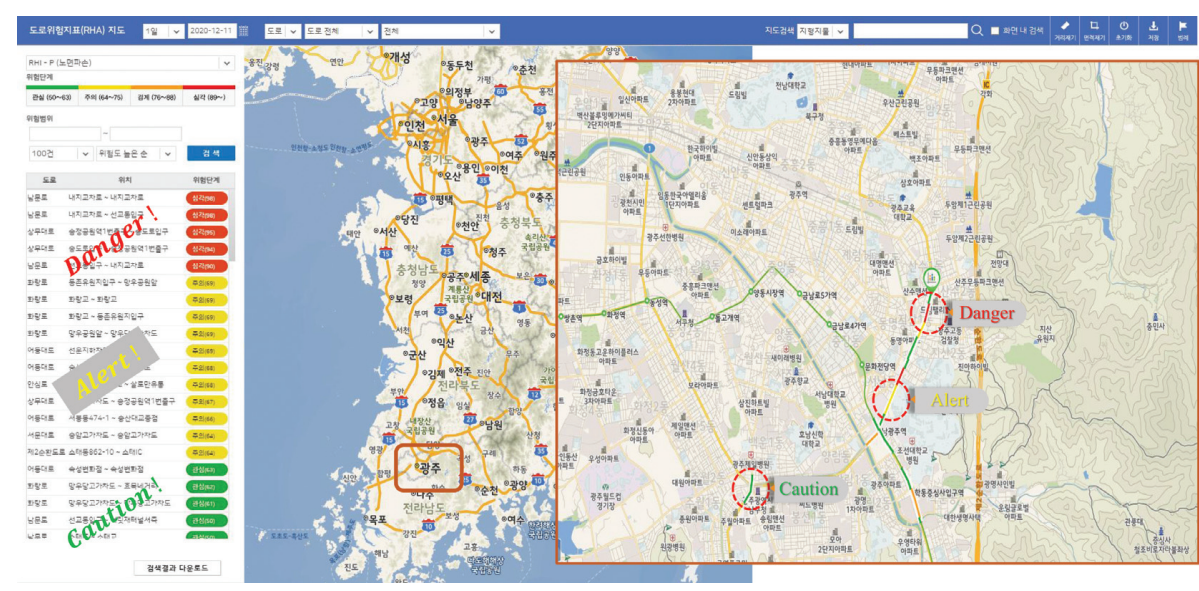

(b)

FIGURE 6: Road hazard index results in Gwangju. (a) Road hazard index results in text format. (b) Road hazard index results in GIS format.

\begin{tabular}{|c|c|c|c|c|c|c|c|c|c|c|c|}
\hline No & Date & Link ID & Section & $\lambda_{\mathrm{pd}}$ & $C V_{\text {pasing }}$ & $C V_{\text {patholdedecting }}$ & \begin{tabular}{|l|}
$\lambda_{\text {pd }} \times$ traffic_volume \\
\end{tabular} & FRD & $Z$ & RHI-P & RHI-Index \\
\hline 1 & 20201207 & 1550043900 & Dongbukro & 1 & 2 & 2 & 35103 & 35103 & 3.1754 & 98.2668 & 4 \\
\hline 2 & 20201207 & 1540007600 & Dongbukro & 1 & 2 & 2 & 35103 & 35103 & 3.0845 & 98.2212 & 4 \\
\hline 3 & 20201207 & 1510003702 & Dongbukro & 1 & 1 & 1 & 35103 & 35103 & 3.0151 & 98.1864 & 4 \\
\hline 4 & 20201207 & 1510004400 & Dongbukro & 1 & 1 & 1 & 35103 & 35103 & 3.0151 & 98.1864 & 4 \\
\hline 5 & 20201207 & 1510049000 & hwarangro & 1 & 1 & 1 & 35103 & 35103 & 2.846 & 98.1017 & 4 \\
\hline 6 & 20201207 & 1510049700 & hwarangro & 1 & 1 & 1 & 35103 & 35103 & 2.846 & 98.1017 & 4 \\
\hline 7 & 20201207 & 1540005000 & Dongbukro & 1 & 1 & 1 & 35103 & 35103 & 2.846 & 98.1017 & 4 \\
\hline 8 & 20201207 & 1510028300 & hwarangro & 1 & 1 & 1 & 35103 & 35103 & 2.6667 & 98.0119 & 4 \\
\hline 9 & 20201207 & 1510030800 & hwarangro & 1 & 1 & 1 & 35103 & 35103 & 2.6667 & 98.0119 & 4 \\
\hline 10 & 20201207 & 1510049100 & hwarangro & 1 & 1 & 1 & 35103 & 35103 & 2.6667 & 98.0119 & 4 \\
\hline 11 & 20201207 & 1510049900 & hwarangro & 1 & 1 & 1 & 35103 & 35103 & 2.6667 & 98.0119 & 4 \\
\hline 12 & 20201207 & 1510050000 & hwarangro & 1 & 1 & 1 & 35103 & 35103 & 2.6667 & 98.0119 & 4 \\
\hline 13 & 20201207 & 1510023400 & Ansimro & 1 & 1 & 1 & 35103 & 35103 & 2.4749 & 97.9158 & 4 \\
\hline 14 & 20201207 & 1510023500 & Ansimro & 1 & 1 & 1 & 35103 & 35103 & 2.2678 & 97.812 & 4 \\
\hline 15 & 20201207 & 1510042200 & hwarangro & 1 & 1 & 1 & 35103 & 35103 & 2.2678 & 97.812 & 4 \\
\hline 16 & 20201207 & 1510028600 & hwarangro & 1 & 1 & 1 & 35103 & 35103 & 2.1409 & 97.7484 & 4 \\
\hline 17 & 20201207 & 1510049800 & hwarangro & 1 & 1 & 1 & 35103 & 35103 & 1.8974 & 97.6264 & 4 \\
\hline 18 & 20201207 & 1510042300 & hwarangro & 1 & 1 & 1 & 35103 & 35103 & 1.7889 & 97.5721 & 4 \\
\hline 19 & 20201207 & 1510045402 & hwarangro & 1 & 1 & 1 & 35103 & 35103 & 1.6202 & 97.3291 & 4 \\
\hline 20 & 20201207 & 1770041600 & Dokripro & 1 & 1 & 1 & $\begin{array}{ll}33279 \\
\end{array}$ & 33279 & 1.5 & 96.5014 & 4 \\
\hline 21 & 20201207 & 1510058700 & hwarangro & 1 & 1 & 1 & 35103 & 35103 & 1.4751 & 96.3301 & 4 \\
\hline 22 & 20201207 & 1510003801 & Dongbukro & 1 & 1 & 1 & 35103 & 35103 & 1.4491 & 96.1511 & 4 \\
\hline 23 & 20201207 & 1510033100 & Dongburo & 1 & 1 & 1 & 35103 & 35103 & 1.291 & 95.0619 & 4 \\
\hline 24 & 20201207 & 1510033300 & $\begin{array}{l}\text { Dongburo } \\
\end{array}$ & 1 & 1 & 1 & 35103 & 35103 & 1.2613 & 94.788 & 4 \\
\hline 25 & 20201207 & 1750004701 & Palmundaero & 1 & 1 & 1 & 13032 & 13032 & 1.2613 & 94.788 & 4 \\
\hline
\end{tabular}

(a)

Figure 7: Continued. 


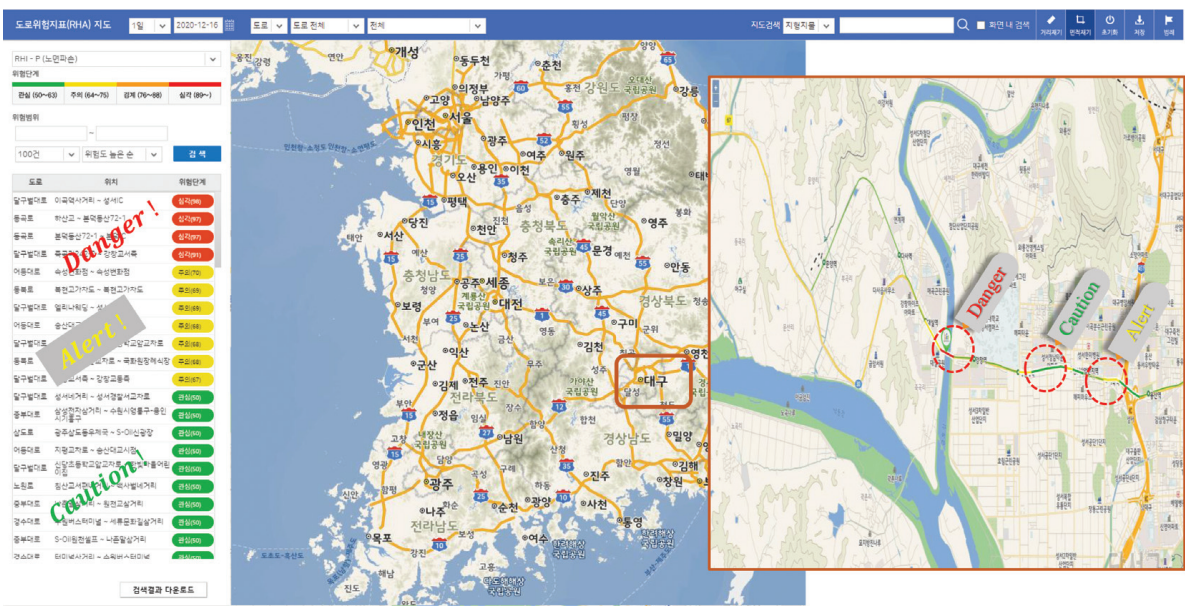

(b)

Figure 7: Road hazard index results in Daegu. (a) Road hazard index results in text format. (b) Road hazard index results in GIS format.

Gwangju were located in urban areas, there was no significant difference in traffic volume between peak and nonpeak hours. Moreover, the average travel speeds were relatively constant. However, test roadway sections in Daegu included an arterial road that connects urban areas to suburban areas. Thus, there was considerable variability in the average travel speed according to non-peak and peak hours. As seen in Figure 6, many sections showed scores between 90 and 100, which implies "danger" level (red color). Likewise, both the field survey and interviews helped identify the reliability of the generated road hazard index.

Indeed, during the test period in Gwangju, the average travel speed was distributed between 45 and $55 \mathrm{~km} / \mathrm{h}$, which is above the speed limit but still relatively slow. Additionally, the traffic volume was relatively high. Furthermore, because these test roadway sections were in a downtown area, there was no significant difference in traffic volume between peak and non-peak hours. On the other hand, the average travel speed in Daegu was distributed between 60 and $90 \mathrm{~km} / \mathrm{h}$, and there were notable differences in traffic volume between peak and non-peak hours.

The results of synthesizing the preferences of the public agencies in charge of the test roads and the field survey to confirm the generated road hazard index from DAISS indicated that the proposed algorithm and final road hazard index accurately reflected the level of risk due to road surface damage.

\section{Concluding Discussion}

Various efforts have been made in the field of traffic safety to provide high-quality traffic information to road operators and general drivers. Such efforts have yielded remarkable achievements through intelligent transport systems (ITSs). As communication and automotive technologies advance, cooperative ITSs (C-ITSs) have emerged to offer better quality and enhanced service levels. Thus, the cooperation between two or more intelligent transport subsystems in personal, vehicle, roadside, and central implementations enables larger ITS services. With the recent shift from ITS to C-ITS in South Korea, the core of ITS is changing from traffic information to traffic safety information.

The main focus of C-ITS is to proactively and quickly identify dangerous road situations/conditions and bring them to the attention of road agencies and general travelers. This article showed how positive benefits to traffic safety can be realized by considering both road surface damage and travel speed data. These data can also be used as a critical source for autonomous driving and to support future C-ITS implementations. The contribution of this study can be summarized as follows:

(i) The results of this study promote the shift from using fixed roadside infrastructure to using mobile infrastructure to collect a variety of traffic information.

In the past, fixed roadside infrastructure was used to collect diverse traffic information from specific points along a certain roadway that can identify hazards and accidents. The algorithm proposed in this study provides a risk level for each roadway section using pothole data detected by in-vehicle smartphone cameras and accelerometers as well as historical average travel speed data. This can potentially reduce fixed roadside infrastructure costs incurred by the public sector and enable data collection along the entire roadway with relatively low maintenance and management costs. This capability is applicable to more advanced vehicle-to-everything (V2X) technologies and has a high potential for commercialization.

(ii) This study introduces the application of artificial intelligence (AI) and big data technologies to traffic safety.

This study demonstrated that AI techniques based on image recognition can be efficiently applied to 
traffic safety analyses. Images of potholes are detected in real time using in-vehicle smartphone cameras employing image analysis software. This enables the collection and processing of a much larger quantity of data compared to conventional methods such as computational or statistical techniques in the traffic safety field. It was also verified that road safety can be improved by publicly disclosing the road inventory data, which can be used by public agencies for more than maintenance purposes. Thus, this study developed and implemented a technology for generating new road hazard information.

(iii) The system proposed in this study improves information redundancy.

There are both predictable and unpredictable situations on the roadways of Korea. Furthermore, traffic flow changes over time and driving environments become increasingly complicated. Therefore, relying on only one or two information sources makes it difficult to ensure safety and expand warning capabilities. The road hazard information derived from the method proposed in this research can overcome such limitations by connecting public data with private traffic information sources. Collecting redundant information from multiple sources enhances the triangulation of results and benefits both the public and private sectors.

As automobiles, sensors, and data processing technology advance, the driving environment is bound to become more complex. It is therefore important to provide real-time information that can account for changes in various driving conditions over time. This study proposed a methodology for quantifying the risk related to road surface damage using vehicle-collected pothole data and database-sourced travel speed data. As data redundancy is crucial in fast-moving environments, the proposed methodology provides additional sources for such data, helping to improve road safety.

\section{Data Availability}

The pothole and road inventory data used to support the findings of this study have not been made available because of data ownership issues.

\section{Conflicts of Interest}

The authors declare that they have no conflicts of interest.

\section{Acknowledgments}

This study was supported by a Korea Agency for Infrastructure Technology Advancement (KAIA) grant funded by the Korean Government (no. 20POQW-B148886-03, Commercial Vehicle-Based Road and Traffic Information System).

\section{References}

[1] K. Kanchanasut, S. Boonsiripant, A. Tunpan, H. K. Kim, and M. Ekpanyapong, "Internet of cars through commodity V2V and V2X mobile routers: applications for developing countries," KSCE Journal of Civil Engineering, vol. 19, no. 6, pp. 1897-1904, 2015.

[2] A. Kulkarni, N. Mhalgi, S. Gurnani, and N. Giri, "Pothole detection system using machine learning on android," International Journal of Emerging Technology and Advanced Engineering, vol. 4, no. 7, pp. 360-364, 2014.

[3] K. Zoysa, C. Keppitiyagama, G. P. Senevirante, and W. Shihan, "A public transport system based sensor network for road surface condition monitoring," in Proceedings of the 2007 Workshop on Networked Systems for Developing Regions, pp. 1-6, Kyoto, Japan, August 2007.

[4] Korea Expressway Corporation, Accident Status Data, Korea Expressway Corporation, South Korea, 2020.

[5] Seoul Metropolitan Authority, Traffic Accidents Statistics, Seoul Metropolitan Authority, Seoul, South Korea, 2020.

[6] R. Brunauer and K. Rehrl, "Supporting road maintenance with in-vehicle data: results from a field trial on road surface condition monitoring," in Proceedings of the 2016 IEEE 19th International Conference on Intelligent Transportation Systems (ITSC), Rio de Janeiro, Brazil, November 2016.

[7] Y. Jo and S. Ryu, Pothole Detection Based on Video Motion Information, pp. 21-23, The Korean Institute of Information Scientists and Engineers, South Korea, 2015.

[8] Y. Jo and S. Ryu, "Pothole detection system using a black-box camera," Sensors, vol. 15, no. 11, pp. 29316-29331, 2015.

[9] T. Kim and S. Ryu, "Review and analysis of pothole detection methods," Journal of Emerging Trends in Computing and Information Sciences, vol. 5, no. 8, pp. 603-608, 2014.

[10] C. Pena-Caballero, D. Kim, A. Gonzalez, O. Castellanos, A. Cantu, and J. Ho, "Real-time road hazard information system," Infrastructures, vol. 5, no. 9, p. 75, 2020.

[11] S. Li, C. Yuan, D. Liu, and H. Cai, "Integrated processing of image and GPR data for automated pothole detection," Journal of Computing in Civil Engineering, vol. 30, no. 6, 2016.

[12] T. Kim and S. Ryu, "Pothole DB based on 2D images and video data," International Journal of Emerging Trends in Computing and Information Sciences, vol. 5, pp. 527-531, 2014.

[13] C. Chun and S.-K. Ryu, "Road surface damage detection using fully convolutional neural networks and semi-supervised learning," Sensors, vol. 19, no. 24, p. 5501, 2019.

[14] T. Lee, C. Chun, and S.-K. Ryu, "Detection of road-surface anomalies using a smartphone camera and accelerometer," Sensors, vol. 21, no. 2, p. 561, 2021.

[15] Korean Institute of Civil Engineering and Building Technology (KICT), "Commercial vehicle-based road and traffic information system," 1st Annual Report, Korean Institute of Civil Engineering and Building Technology (KICT), Goyangsi, South Korea, 2018.

[16] Korean Institute of Civil Engineering and Building Technology (KICT), "Commercial vehicle-based road and traffic information system," 2nd Annual Report, Korean Institute of Civil Engineering and Building Technology (KICT), Goyangsi, South Korea, 2019.

[17] I. Malygin, V. Komashinskiy, and O. Korolev, "Cognitive technologies for providing road traffic safety in intelligent transport systems," Transportation Research Procedia, vol. 36, pp. 487-492, 2018. 
[18] Korean Institute of Civil Engineering and Building Technology (KICT), "Commercial vehicle-based road and traffic information system," 3rd Annual Report, Korean Institute of Civil Engineering and Building Technology (KICT), Goyangsi, South Korea, 2020.

[19] C. H. Yang and A. C. Regan, "A multi-criteria decision support methodology for implementing truck operation strategies," Transportation, vol. 40, no. 3, pp. 713-728, 2013.

[20] C. H. Yang, A. C. Regan, and I. S. Kim, "Estimating road management equipment inventory needs and associated purchase costs," Transport Policy, vol. 36, pp. 242-247, 2014.

[21] Korean Association for Organizational Studies, (KAOS), Analysis of the Effect of Introducing and Operating Information Systems within the Government, Ministry of the Interior and Safety, Jongno, Seoul, South Korea, 2010.

[22] C. Yang and J. Kim, "Developing road hazard estimation algorithms based on dynamic and static data," The Journal of The Korea Institute of Intelligent Transport Systems, vol. 19, no. 4, pp. 55-66, 2020.

[23] C. Yoon and J. Sung, "Development of Integrated highway management system in Korea," Proceeding of the Eastern Asia Society for Transportation Studies, vol. 5, pp. 783-790, 2005. 